\title{
INTELLIGENT ROBOTIC ARM
}

\author{
Prasad Ban ${ }^{1}$, Shweta Desale ${ }^{2}$, Revati Barge ${ }^{3}$, Pallavi Chavan $^{4}$ \\ ${ }^{1}$ Information Technology, Ramrao A dik Institute of Technology, Nerul, Navi M umbai, M H, India \\ 2Information Technology, Ramrao A dik Institute of Technology, Nerul, Navi M umbai, M H, India \\ 3Information Technology, Ramrao A dik Institute of Technology, Nerul, Navi M umbai, M H, India \\ ${ }^{4}$ Information Technology, Ramrao A dik Institute of Technology, Nerul, $\mathrm{Navi}$ M umbai, $\mathrm{M} \mathrm{H}$, India
}

\begin{abstract}
Regardl ess the abundance of technological robotic equipment's present in the form of auto- mated M echanical Robotic A rm, most of the citizens in India are not able to use those robots in their regular day to day life. This paper talks about the Intelligent robotic A rm (IRA) which is able to cook food dishes in an ordinary home environment. The robotic arm recognizes the utensils and ingredients required for the particular recipe. For object detection we have developed a system using 0 penCV and Python which helps the robotic arm to recognize the correct ingredient and pick and drop it to appropriate utensil for cooking the required recipe. It involves automated tasks which the arm has to perform while commands are given to it for making the particular recipe. Our motivation is to help brilliant supplier robots that work by and large human environments, automating continuous errands. In quest for this objective, we follow the universal apply autonomy worldview, in which savvy observation and control, are blended in with ubiquitous processing. By misusing sensors and effectors in its condition, a mechanical can work extra confused obligations other than turning out to be excessively perplexing itself. Following this understanding, we have built up a supplier automated that works independently in a sensor-prepared kitchen. The mechanical gains from exhibition, and performs refined assignments, working together with the system of gadgets in its condition. We record on the structure, usage, and utilization of this framework, which is unreservedly accessible for use, and improvement through others, in the examination network .
\end{abstract}

\section{INTRODUCTION}

Detecting objects and specific elements in real world by any robot itself is still a crucial task. Robots have the capability to work precisely and in almost $97 \%$ accurate form. Real time object recognition entails identification of objects by means of Computer V ision and A rtificial Intelligence, it involves M achine L earning and Deep L earning concepts. Intelligent Robots can be used in day to day life of a normal middleclass person in India in fact the main objective behind the making of robots is to reduce the physical workload on Human, to automate the completion of tasks and to get more precise and accurate outcomes. [1] The system proposed is that automating and assisting in regular cooking work in a kitchen of a normal middle-class person in India by use of a Robotic Arm. Thus, the problem we define here is to design and develop an intelligent $M$ echanical Robotic $A$ rm which could be used in regular home environment for the purpose of cooking meal. The system itself would be able to cook normal recipe in kitchen via a remote access to the robotic arm through a smartphone. Rather than commonplace mechanical mechanization frameworks robot ought to have the option to respond to unforeseen, questionable and obscure occasions by parameter adjustment or extra arranging exercises. The robot@ capacity to gain from its $\mathrm{n}$ experience and through human direction is done through Al. Learning should prompt quicker issue arrangements, quicker or progressively solid arrangement executions or to the capacity to take care of issues the robot was not ready to comprehend previously. Profound learning permits robots to improve examination and recognition of unvulgar things through progressively definite and complex information. Our base approach to achieve the goal includes [a]developing a low budget affordable robotic arm and [b] system capable of recognizing and detecting objects and ingredients used in cooking meal with the use of Image Processing, Computer vision, A rtificial Intelligence and $M$ achine Learning. [c] Robotic arm capable of picking and dropping the ingredients recognized by the system. Imagine you are being offered a meal which has been taught by you to the robot, isn't it would be a great pleasure for you? This section describes the background in brief and gives the reader an overview of the problem and a better understanding of what artificial intelligence, robotics, machine learning and deep learning technology is and why it is needed. A rtificial intelligence is the most growing technology. The real-world data is collected and the robotics are trained with that data through artificial intelligence technology. Robotics are manipulated with that real-world data, more the data is used, more will be the performance of robot improves. Along these things by consolidating man-made brainpower, $\mathrm{Al}$ and profound learning perspectives, the completely mechanized and shrewd robot can prepare home-made supper with no human intercession. It will learn plans and cook them for you.

\section{LITERATURE REVIEW}

The overall market changes when new technology is introduced. The technologies help speed up the processes and bring more efficiency into the market. Cooking takes a ton of time. Somebody in the family winds up going through a few hours in the kitchen every day. This ends up being an exceptionally substantial weight on family units and unhitched males/old maids. The Robotic Chef helps the weight of the cook by setting up a help in cooking by automated processes. [1] Nowadays many companies are developing robots and R obotic A rms having different use in automating the human tasks but most of the A rms and Robots are only used for industrial purpose and process automations. [2] Companies are also developing R obots for cooking meals in kitchen in replacement for human chef, some of which are being devel oped by M oley R obotics and Samsung R obotics, including a progressed, completely practical robot incorporated into a delightfully structured, proficient robotic kitchen setup. The complete setup is operated by a single interface on a touch screen panel integrated with the robotic setup. M oley has made the world @ first automated kitchen. 
Highlighting a progressed, completely practical robot incorporated into a delightfully structured, proficient kitchen, it cooks with the ability and style of an ace culinary expert. The model was debuted to boundless approval at $\mathrm{H}$ anover $\mathrm{M}$ esse, the global mechanical technology appears. [3] A B angal ore based startup also came with a modern solution for the problem of cooking. They devel oped a robot which can prepare a meal when pre-cut vegetables are introduced to it. Its user only needs to load in cut vegetables. The machine does the everything else and presents a delicious meal. This Cooking R obot makes numerous Indian Foods by doing 3 operations. One is, dropping - fixings into the dish being cooked; Second operation is, Heating - the dish being cooked; The third one is, Stirring - the substance. These three activities are everything necessary to get ready numerous Indian dishes. The machine as of now comes stacked with all the flavors that are required for cooking the client includes the vegetables required for the cook and the machine at that point dominates and does everything else. At the point when the client chooses what they need to cook, the machine guides them through what vegetables they have to get ready and cut, in the amounts they need. It at that point guides them through the stacking of the vegetables into the machine. The vessels that hold the vegetables and any piece of the machine that comes into contact with the nourishment can be snapped here and there, and is in this manner simple to clean. The quantity of such parts to clean is additionally limited. Individuals will incline tow ard the adaptability of having a cooking machine within reach that can cook for them whenever, particularly one that they can control from their telephone so it begins cooking when its clients are still on their way once more from work. In spite of the fact that this Robot does a large portion of the Cooking consequently, it despite everything has a few confinements. W ith the present A I Technology, it is as yet not a basic issue to fabricate cutting machines that are likewise simple to clean. A long these, a human would be expected to do the cutting and stripping. The M echanical Chef likewise doesn (Eset up any dishes that require molding, for example, dosas and idli and the size of this robot is huge as it won't fit into an average sized kitchen. [4] A nother one of the best inventions which we studied is D orna robotic arm. Dorna is a quick, amazing and exact automated arm. Dorna is a modern evaluation 5-hub mechanical arm with an open source programming and firmware, and it is equipped for computerizing numerous genuine assignments. Dorna gives an ideal answer for entrepreneurs, producers, analysts, specialists and any individual who needs to quit fooling around in mechanical autonomy without spending a fortune on a modern automated arm, but again the basic problem with this arm is that Dorna is not affordable for small scale earning people.[6]A Igorithms of Computer V ision and Deep L earning modules which are able to find and classify objects in the live feed from PiCam and normal USB-Camera. Some of the algorithms useful for Deep Learning, Canny Edge algorithm in OpenCv and the CV2 libraries, CUPA toolkit for deep learning (Developed by NVDIA). [4] Canny Edge and other imutils Python libraries with CNN and TensorFlow have been successfully implemented for object detection in O penCV. [5] The reason behind selecting this algorithm is that it is compatible with python libraries in OpenCV and works efficiently on Raspbian Operating System. PC have gigantic learning ability and have exhibited predominant insight in numerous spaces. Cooking Robots could be helpful to the individuals with serious physical incapacity. In this way, cooking includes numerous troublesome undertakings that requires physical connection like cutting, and so forth. Hence, it is as yet a moving activity for a robot to perform in typical kitchen condition. L ate research have improved the robot@ ability what $@$ more, adaptability to $P C$ vision and arranging. The utilitarian item situated system (FOON) to demonstrate the availability of the utilitarian related items and their movements in control undertakings. The graphical model FOON is learned by watching object state change and human controls with the objects. Utilizing a very much prepared FOON, robots can decode an errand objective, look for the right objects at the ideal states on which to work, and create a succession of legitimate control movements. A chart recovery approach is introduced to produce control movement groupings from the FOON to accomplish an ideal objective, showing the adaptability of FOON in making a novel and versatile methods for taking care of an issue utilizing information assembled from numerous sources. The outcomes are shown in a reproduced domain to outline the movement successions created from the FOON to complete the ideal undertakings. To remove information from instructional recordings , a four - arrange video understanding pipeline is created. This assistance to perceive all occasions in video and play out the total errand as needs be.[6] The double arm humanoid cooking robot can cook dishes like an ace culinary specialist in common home kitchens. The kinematic investigation is first done for the 6DOF arm of the robot. Organize outlines are relegated to each connection of the arm utilizing the Denavit-Hartenberg show. The dynamic conditions are then acquired utilizing the Lagrangian technique by ascertaining motor vitality and potential vitality. A reenactment for a Chinese cooking devel opment is then done, joint torques for each joint of the robot $@$ arm during this development are obtained. Recent inquire has been made to bring the PC insight into physical world with the goal that robots can be capable perform physical control assignments. The approach first enable robots to become familiar with the aptitudes by "viewing" on the web instructional recordings. Subsequent to looking out for 200 recordings the expertise to adjust PC knowledge is created.[7] Sereneti needs Cooki to be a piece of an environment that incorporates an assistance called Foodie, where they send you pre-bundl ed fixings that your robot at that point cooks for you. At this moment, Cooki can deal with up to four fixings, and the last rendition will likewise incorporate a ring over the container to include oil, salt, pepper and other spices.lt $\$$ additionally be littler and particular with the goal that you can extend number of ingredients.Sereneti was focusing on a mid 2017 release.

\section{INTELLIGENT ROBOTIC ARM}

This paper proposed a simple robotic arm which is a 6 d. o.f arm (having 6 degree of freedom). . . The main model of the robotic arm comprises the basic three components. [a]A ctuators (Servos), [b]End Effector, [c]Controller (Arduino). The actuators are responsible for the movement of the robotic arm in the tri A xis coordinates. End effector is the component which grips the object which is to be picked up and the brain of the arm, the controller it controls all the movement of the complete robot and the actuators.

\subsection{System Architecture}

A rchitecture of the project provides the complete Insight of the project; this alludes the complete structure of the software and control of creating such logical and systematic structures. System architecture of this project is in figure 1. 


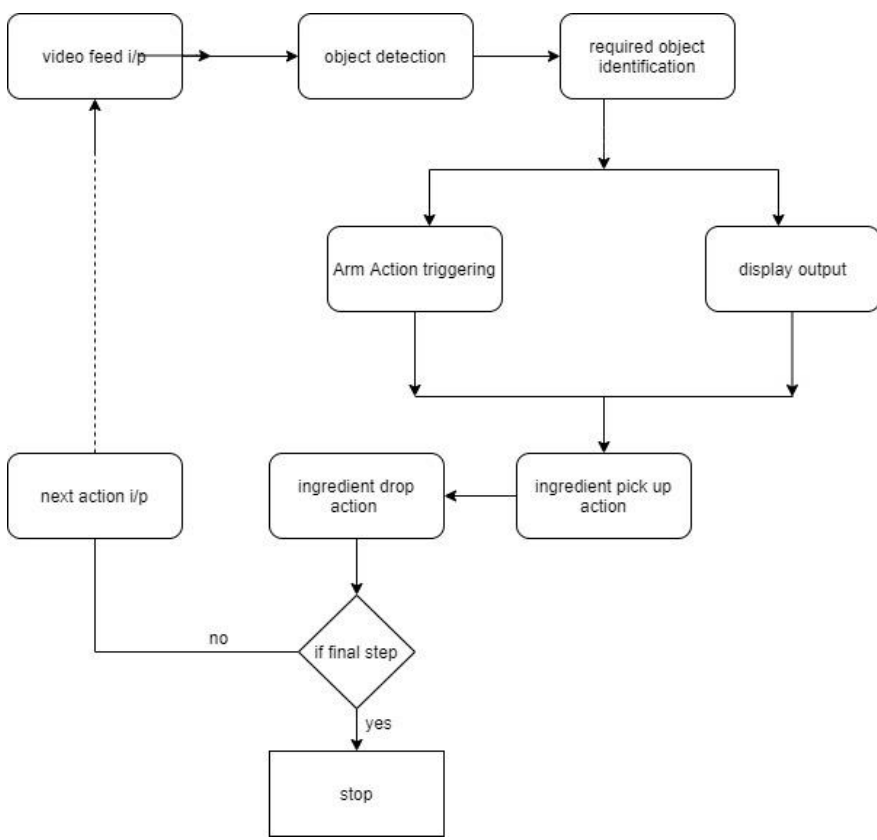

Figure 1. architecture of the proposed system

The Development process is designed in following steps:

- Live feed from PiCam to system

- Object Detection and ingredient identification

- Setting the particular recipe

- $\quad$ Pick and drop action by Robotic A rm

Input to the system includes the object detection and identification of the required ingredient which is first required for preparation of the particular recipe. The image processing Algorithm used for the proposed system works on LeN et - CNN (Convolutional Neural Network) and TensorFlow. The system takes live feed through the PiCam and matches the similar objects which are being found through the input using Canny-E dge algorithm for object identification. Science all edge detection outputs are easily affected by noise, to prevent the false detection a gaussian filter kernel is bonded to the image, the equation of gaussian filter kernel smoothening is given by:

$$
H_{i j}=\frac{1}{2 \pi \sigma^{2}} \exp \left(-\frac{(i-(k+1))^{2} \dashv \cdot(j-(k+1))^{2}}{2 \sigma^{2}}\right) ; 1 \leq i, j \leq(2 k+1)
$$

In post processing, the output from the live feed i.e. identified ingredient triggers a signal to the script which initiates the robotic arm to pick up the particular ingredient and drop it at a particular destination which is already set. Further the mapping of the code written for particular recipe is done and next actions are taken which includes again scanning for the next ingredient required.

\subsection{IMPLEMENTATION}

The proposed model is a R obotic arm which is operated by A rduino UNO and Raspberry Pi for cooking which is built in low cost. The Robotic arm is capable for cooking recipe in regular home environment, for example: for the set of code written for the recipe of normal $M$ aggie, after triggering the recipe for $\mathrm{M}$ aggie the $\mathrm{O}$ bject detection algorithm will be searching for noodles and $M$ aggie spices and after detecting the required ingredients the arm will pick up the ingredients and drop it at the required utensil. [1] 0 bject detection and recognition is a technique to change over a video image for recognizing the particular element in the feed. For successful working of the algorithm we need to feed the model and compile a huge set of labelled data and train it accordingly.
The object detection is used in many domains where the projects related to computer vision are implemented example, the automated selfdriving car. Object detection also known as computer vision is a technical field that deals with searching the ways to automate all the job that a human visual system can do.[2] Taking deep learning-based object detection concept, there are three basic methods which can be used for the object detection purpose. [a]Faster R-CNN'S [b] Y OL O (you only look once) and the third one is [C] Single shot D etectors. The SSD is developed by the google, SSD method stands more accurate than R-CNN and Y OLO

M obileN et: Efficient deep learning neural network

MobileNet are more compatible for running on small devices like raspberry pi and any smart-phone, these are differentfrom normal CNN because of the usage of the depth wise separable convolution. In the proposed model the base object detection is built using M obileN et SSD and the deep neural network module.

The end result of this method in deep learning-based object detection is that it can process approximately 16-18 fps depending upon the speed of the system being used, and the output is given by checking the confidence (i.e. probability) associated with each detection because there can exist multiple similar object in the input feed provided.

Tensorf low is a free and open-source programming library for dataflow and differentiable programming over a scope of errands. It is a representative math library, and is additionally utilized for AI applications, for example, neural systems. In March 2018, Google declared TensorF low.js adaptation 1.0 for A I in J avaScript. In J an 2019, Google declared Tensorflow 2.0. It turned out to be authoritatively accessible in Sep 2019.In M ay 2019, Google declared TensorFlow Graphics for profound learning in PC graphics. TPU is a programmable A I quickening agent intended to give high throughput of low-exactness number juggling (e.g., 8-piece), and situated toward utilizing or running models as opposed to preparing them. Google declared they had been running TPUs inside their server farms for over a year, and had discovered them to convey a request for size better-advanced execution per watt for A I. E dge TPU is Google@ motivation assembled A SIC chip intended to run TensorFlow Lite AI (ML) models on little customer processing gadgets, for example, smartphones known as edge figuring. among the applications for which TensorF low is the establishment, are computerized picture inscribing programming, for example, Deep Dream. Rank Brain now handles a significant number of search inquiries, supplanting and enhancing conventional static calculationbased inquiry results.

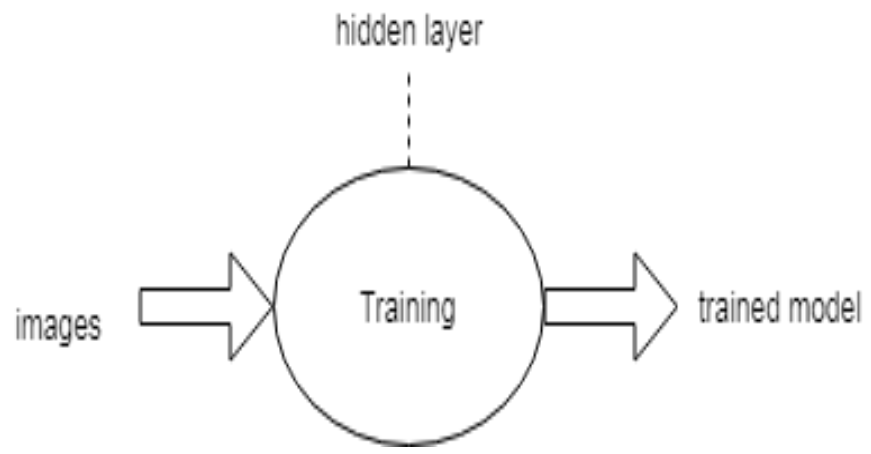

Figure 2 [Training of database]

a neural network is a mind-boggling information esteem and can be put away as a typical item. The object-situated methodology creases the most agreeable and characteristic configuration model for neural systems. In the terms of article arranged database frameworks neural systems are deal t with for the most part as mind boggl ing objects. These frameworks demonstrated truly important at taking care of what $@$ more, 
administrating such articles in various regions, as PC supported structure, geographic databases, organization of part structures, etc. The proposed worldview permits the meaning of a solitary system up to a group of comparable systems. These are systems with comparable properties, similar to organize worldview and structure. The term comparable is not limited to arrange properties just, however covers additionally organizes, which achieve uncommon undertakings. The attributes of these systems can be totally different, yet the database input is the equivalent. The information control offices of the framework are abused to deal with the systems and their assignments inside a similar structure.

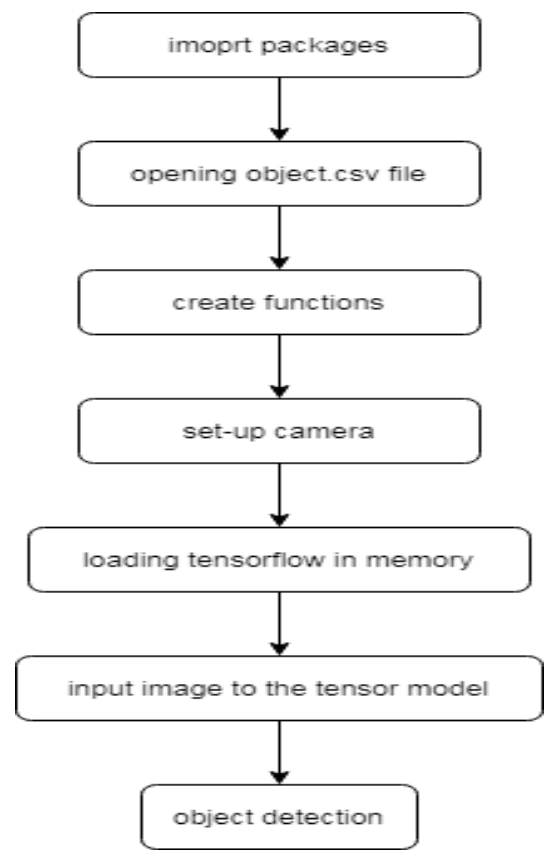

Figure 3 [ TensorFlow Construction]

A neural system (NN), on account of counterfeit neurons called artificial neural system (A N N) or simulated neural system (SNN), is an interconnected gathering of natural or artificial neurons that utilizes a numerical or computational model for data preparing dependent on a connectionistic way to deal with cal culation. M uch of the time an A N N is a versatile framew ork that changes its structure dependent on outside or interior data that courses through the system. In increasingly commonsense terms neural systems are non-linear factual information displaying or dynamic devices. They can be utilized to demonstrate complex connections among information sources and yields or to discover designs in information.

Video enhancement entails removal of the different noise from the video feed. There is a need to boost the qual ity of the live feed which is being analyzed by the al gorithm for the better outcome. The input feed is the live video which is taken by the PiCam or any other digital camera which is connected to the raspberry pi.

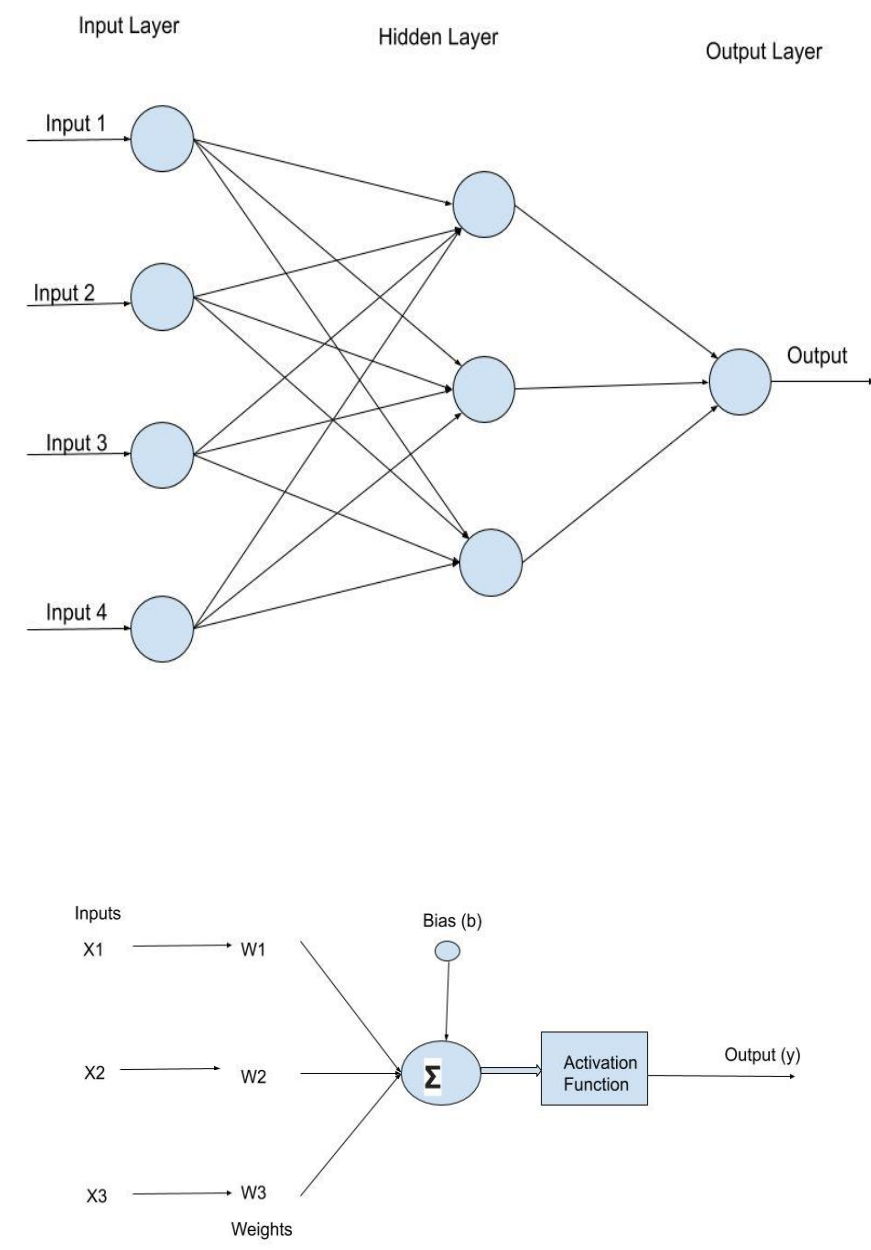

Figure 4 [Feedforward Neural N etwork]

The resol ution of the camera is a considerable factor which captures the live feed. The credibility of the element detection depends on the resolution of the camera used.

The PiCam which we are using is shown in figure 5.

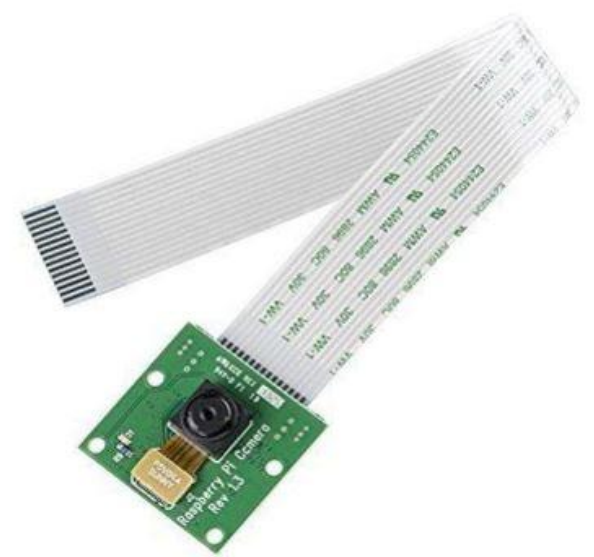

Figure 5. [PiCam] 


\section{Prototype Execution}

The proposed model works on then deep learning mechanism. Deep Learning and neural network-based methods have transcended traditional machine learning techniques by a vast scope in terms of accuracy in many areas of Computer $V$ ision. The object detection is done from the live stream from the PiCam/W ebcam. Depending on the recognized object the controller of the arm initiates the gripping action as they are pre-feded to the system. The End effector which is the gripper of the robotic arm hold the object to be picked up. The end Effector act as the human wrist for the pick and place work. There are numerous end effectors which can be used for the gripping purpose. The one which is used in proposed model in in figure 2.

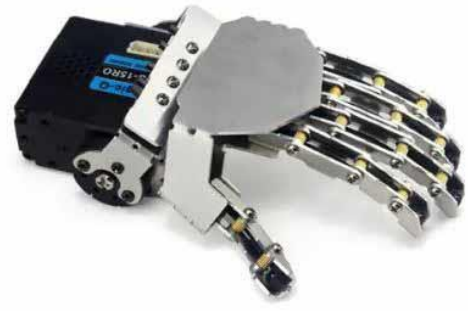

Figure 6. [Gripper]

\section{RESULT}

The input was provided in the form of live feed through the webcam attached at the end of the prototype robotic arm. The al gorithm of object detection applied on the live stream through the web cam which works on 60fps. The object is recognized which is required for the particular search which is to be performed. The model scans the whole region which is under the frame of the webcam and detects the required object/element. The output of the process is that the required element is detected for performing the pick and place task. The prototype model grips the object and uplift it and place it at the desired destination or performs the further task as instructed by the code written for the particular recipe. Following image opl shows how the webcam connected at the terminal of the prototype robotic arm shows the result at the monitor frame.

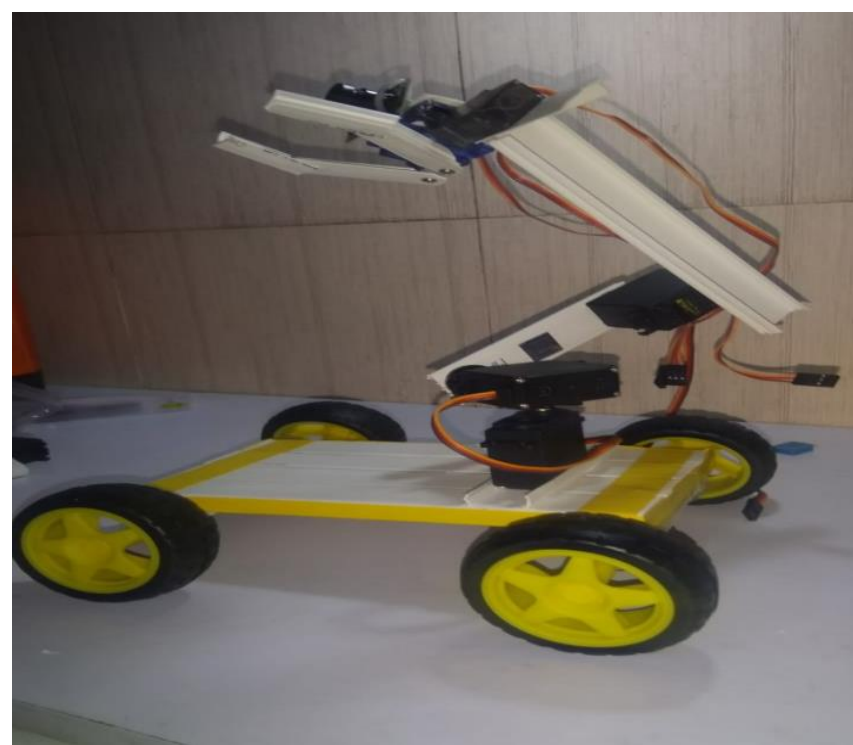

Figure 7- [model for prototype experimentation (without gripper)]
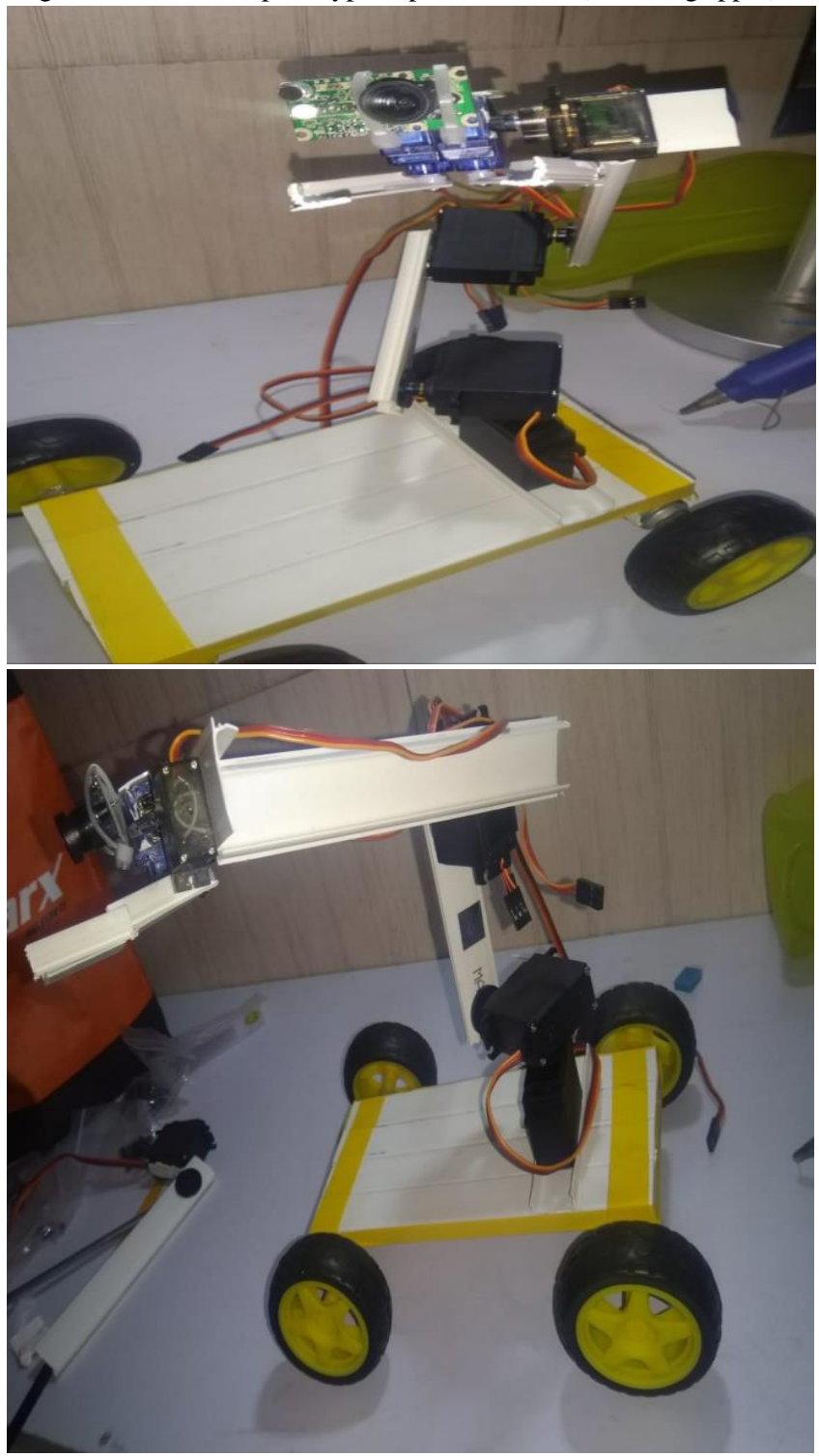

Figure 8 [web cam used for experimentation.]

A fter performing the algorithm for detecting a spoon in the live stream through the web can it showed the following result- 


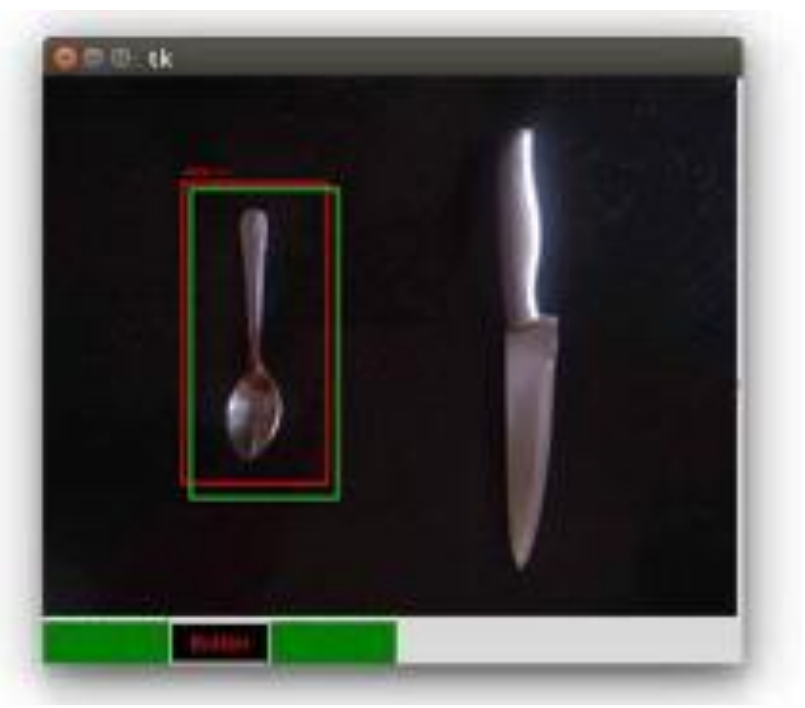

Figure 9 [Object Recognition]

\section{CONCLUSION}

The model automates the cooking process in normal home environment which will play an important role in helping humans to assist in cooking. A fter conducting survey, we found that existing cooking robots are much expensive and not portable. Since the world is rapidly changing and dive loping, there are lot of technological advancements are happening on a daily basis. Therefore, if we have something powerful and less expensive at our disposal then we need to ensure that we make use of it to full extent. A $n$ automatic cooking arm is presented which will fulfill the basic cooking tasks. A cost effective, more efficient and small size intelligent robotic arm will be developed.M oreover, the precision factor of the object detection al gorithm needsto be improved. The proposed model Is not yet developed to perform for longer period on the time, as it will be working in the heat surrounding it need to get protected from the several environmental effects which can cause damage to the robot.The main aim of this system is to cook different recipe without human intervention. So, the future scope to the project is to engross and perform all the operations which arenecessary for the cooking purpose and to get controlled through a remote accessible application through web or mobile phone.

\section{References}

[1] Jiaxin Zhai ; Gen Pan ; Weixin Yan ; Zhuang Fu ; Yanzheng Zhao," Dynamic analysis of a dual-arm humanoid cooking robot ", 2015 IEEE 10th Conference on Industrial Electronics and A pplications (ICIEA), 10.1109/ICIEA .2015.7334226

[2] Oussam Khatib , Journal of Robotics, February 1987, “A Unified A pproach for M otion and Force Control of Robot M anipulators: The Operational Space Formulation", Vol. RA-3, NO. I

[3] Yu Sun, "Robot Perception and Action Lab", Department of Computer Science and Engineering, University of South Florida, USA “ Al Meets Physical World Exploring Robot Cooking” arXiv: $1804.07974 \mathrm{v} \mathrm{I}$

[4] Radu B ogdan R usua, B rian Gerkeyc, M ichael B eetz — "Robots in the kitchen: Exploiting ubiquitous sensing and actuation", Researchgate2018, 10.1016/j.robot.2008.06.010

[5] raspberry-pi robotic arm with computer vision-electrom dust(youtube). https://www.youtube. com/watch? $v=b L 8 f S c d B 00 s \& t$

[6] Timothy Chen, "Cooki, A desktop robotic kitchen chef that does everything, IEEE 2018 [7] Adrian Rosebrock. PyImageSearch" (Deep Learning and Computer Vision)

[8] Samsung bot chef(robotic kitchen by samsung)-cnet.com

[9] Michael Beetz — "Robots in the kitchen: Exploiting ubiquitous sensing and actuation".researchgate, 10.1016/j.robot.2008.06.010

[10]D orna(high performance robot), dorna.ai. 